\title{
DESIGN STUDIES OF AN ELECTROSTATIC STORAGE RING
}

\author{
C. P. Welsch ${ }^{1}$, J. Ullrich, MPI-K, Heidelberg \\ C. Gläßner, K.-U. Kühnel, A. Schempp, IAP, Goethe University, Frankfurt \\ H. Schmidt-Böcking, IKF, Goethe University, Frankfurt \\ Germany
}

\begin{abstract}
Electrostatic storage rings combine a number of very interesting characteristics that make them an attractive tool in the low energy range. In contrast to magnetic rings, all of the fields in an electrostatic storage ring are completely mass independent. At the same particle energy and charge state, ions from light protons to heavy biomolecules can in principal be stored with identical field setups.

A small ring for ions of energies up to $50 \mathrm{keV}$ is planned to be build up at Goethe University in Frankfurt. Different designs have been calculated and the results are presented in this contribution. Furthermore, prototypes of the necessary optical elements have been manufactured and are described as well.
\end{abstract}

\section{INTRODUCTION}

In 1997 the first electrostatic storage ring began operation at ISA, Denmark [1]. Many different types of ions could be stored in the ring ELISA since and the expected flexibility of the machine with respect to storable ions could be proven.

Operating at energies of some $10 \mathrm{keV}$, electrostatic rings can be seen as a cross between 'classical' magnetic rings and electrostatic traps. They offer easy access to all their elements, a wide range of possible experiments at relatively low costs.

In order to improve the resolution in existing experimental setups and to cover new fields, a small machine for total energies up to $50 \mathrm{keV}$ is planned in Frankfurt.

\section{POSSIBLE LAYOUTS}

From the point of view of particle dynamics and because of practical reasons, the symmetry of a storage ring should be chosen as high as possible. This reduces calculations to small fractions of the whole circumference and takes advantage of the structure's periodicity.

Optical elements can be manufactured and utilized several times and the straight sections give plenty of room for experiments. An overview of such a design is shown in Fig. 1.

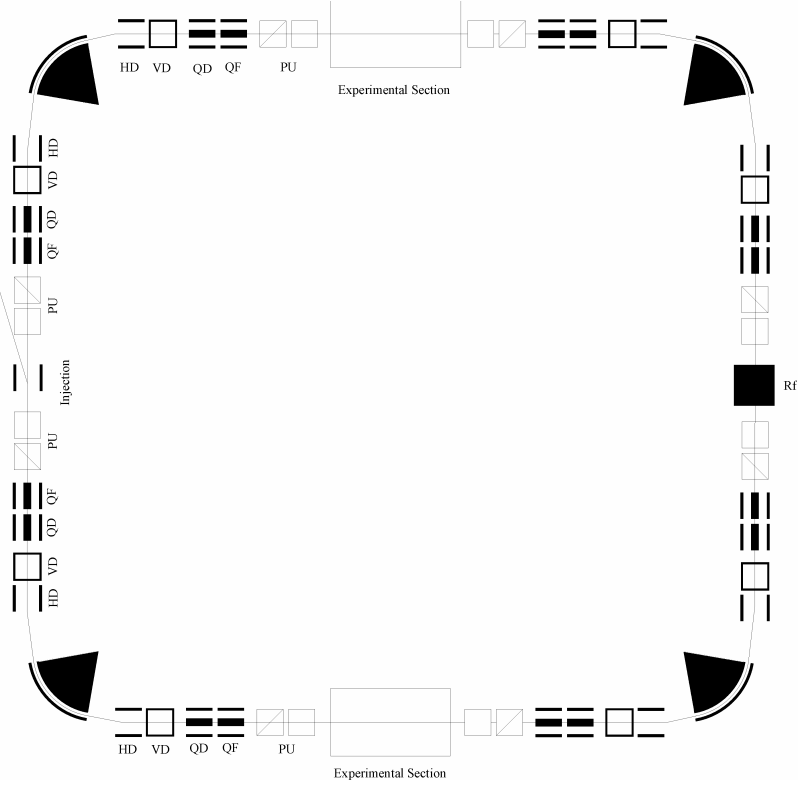

Figure 1: classical "ring" shape

The distances between the different elements are not drawn to scale in order to facilitate a first overview.

Injection into the machine can be done in two different ways: Either the beam enters the ring at a small electrostatic inflector, that bends the beam on axis and is switched off after half the ring's circumference is filled, or it enters along one of the straight section, where one of the corner deflectors would have to be switched off at the beginning.

Another reason for splitting the $90^{\circ}$ corner sections up into three elements is the possibility of bringing e.g. laser or electron beams into the machine and thus enabling interaction experiments.

The beam envelope corresponding to this first design is shown in Fig. 2. A symmetry point lies in the middle of the cylindrical deflector and a smooth propagation through the system is reached. It should be pointed out that in the cases (3) and (4), the beam is focused in this bend in both planes and higher space charge forces occur. 

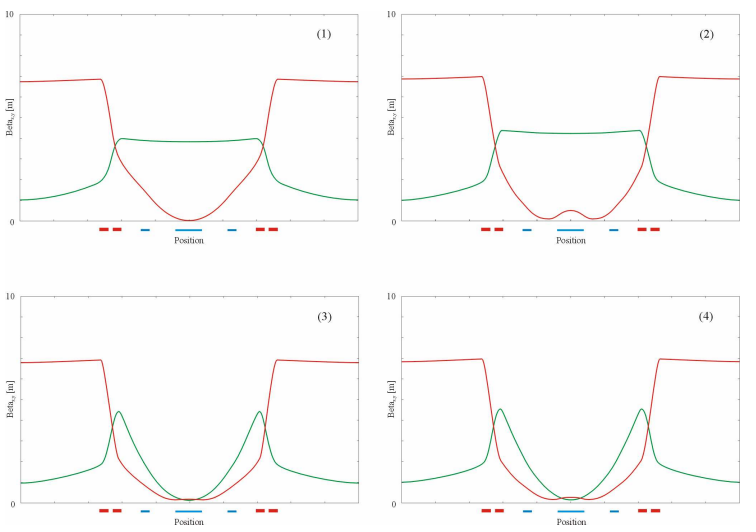

Figure 2: Beta-function for a classic shape

A racetrack shape design as given in Fig. 3 reduces the size of the whole machine even further at the cost of a lower symmetric lattice.

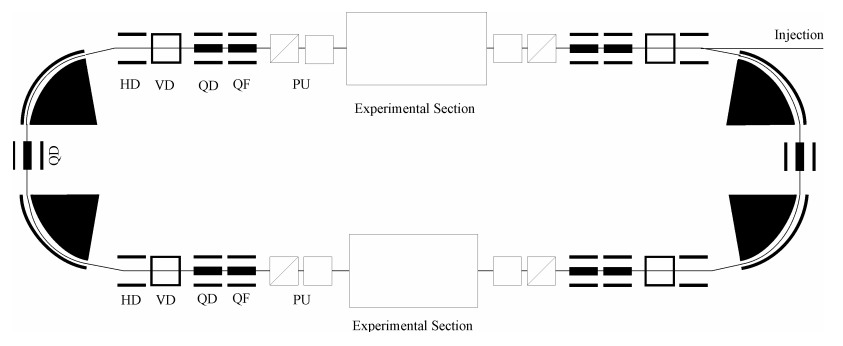

Figure 3: racetrack shaped ring

In this example, the width of the whole machine could be reduced below 2 meters, which would make the complete ring transportable.

The size of the elements was kept identical in order to use the results of previous calculations. The tune values for two different points of stable operation are given in Table 1.

Table 1: Tune values for a racetrack design

\begin{tabular}{||c|c|}
\hline \hline parameter & value \\
\hline$Q_{x}$ & 2.248 \\
$Q_{y}$ & 0.295 \\
$Q_{x}$ & 2.82 \\
$Q_{y}$ & 0.266 \\
\hline
\end{tabular}

Direct comparison of the beta functions in a circular and racetrack like machine show that in the latter case, the beam dimension can be reduced even further.

\section{OPTICAL ELEMENTS}

\subsection{Deflecting elements}

For the different reasons mentioned above, the $90^{\circ}$ bend in the corners of the ring is split up into three elements.

A first small angle deflection is done in a parallel plate deflector. A set of two bends is put in one cavity as shown in Fig. 4. One of electrode pairs is rotated by $90^{\circ}$ and is used for closed orbit correction.

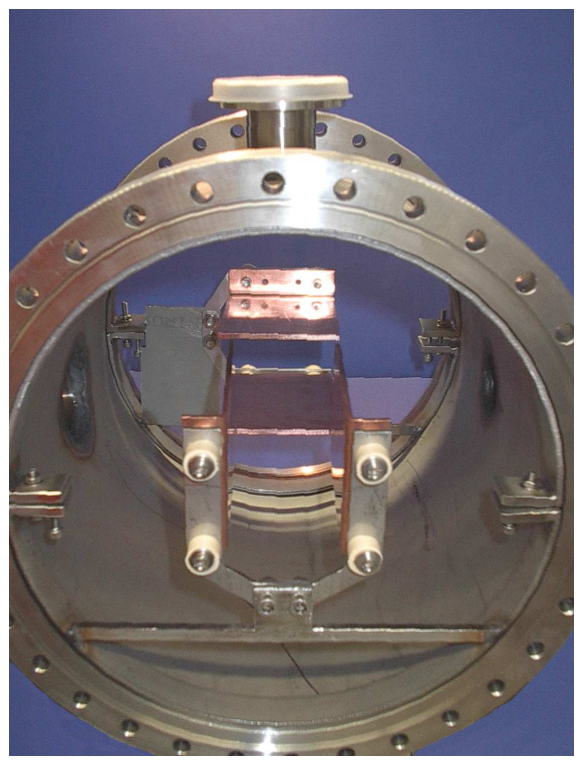

Figure 4: $10^{\circ}$ parallel plate deflector

Necessary voltages are about $\pm 4.5 \mathrm{kV}$ per electrode at plate distances of $100 \mathrm{~mm}$.

The main bending of $70^{\circ}$ is done in a cylindrical deflector as shown in Fig. 5. At the entrance and exit of this element grounded shields are placed in order to reduce the effects of fringe fields [3].

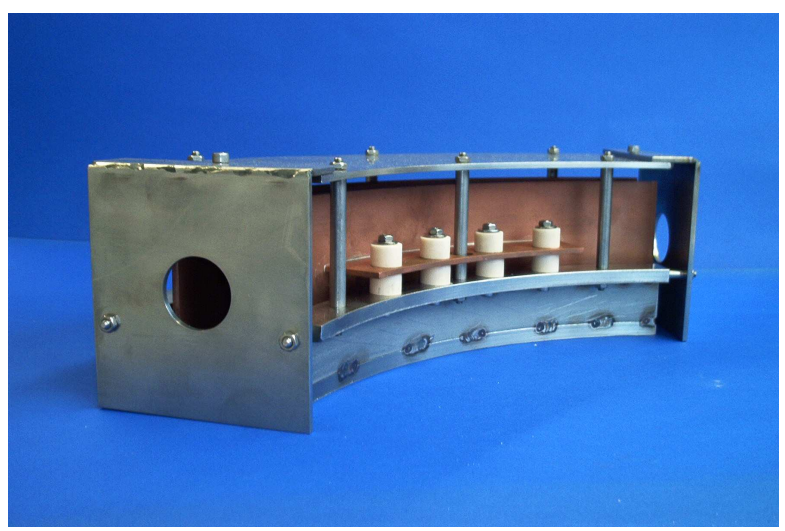

Figure 5: $70^{\circ}$ cylindrical deflector 


\subsection{Transverse Size}

It is only possible to cover the requirements of different experiments if the beam size and shape can be changed within a wide range. For that purpose, several pairs of quadrupole lenses are foreseen. From a strongly focussed to a parallel beam, many shapes are possible.

Each electrode is $10 \mathrm{~cm}$ long and the inner aperture is roughly $2.5 \mathrm{~cm}$.

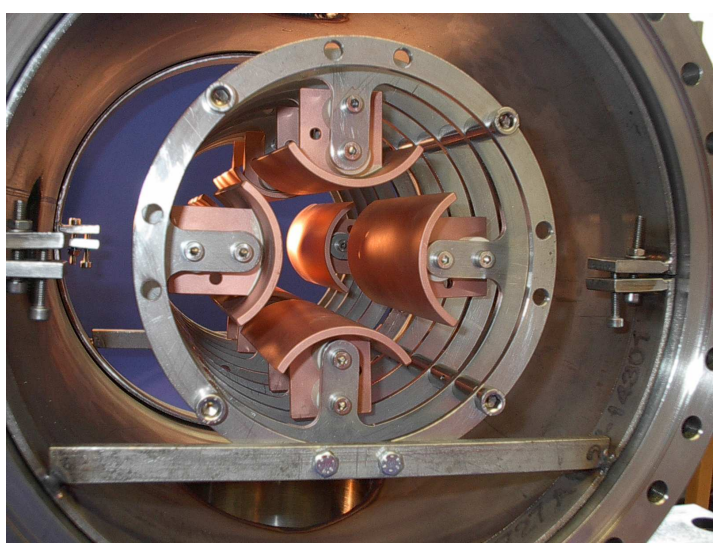

Figure 6: $70^{\circ}$ cylindrical deflector

A list of the latest design parameters for a circular layout is given in Table 2 .

Table 2: List of design parameters

\begin{tabular}{||l|r||}
\hline General Parameters & $50 \mathrm{keV}$ \\
\hline Maximum energy & $17.91 \mathrm{~m}$ \\
Circumference & $3.5 \mu \mathrm{s}(\mathrm{p})$ \\
Revolution time & \\
$10^{\circ}$ deflectors & \\
\hline Plate area & $100 \mathrm{~mm}$ \\
Plate distance & $+/-4.5 \mathrm{kV}$ \\
Voltage & \\
$70^{\circ}$ deflectors & $70 \mathrm{~mm}$ \\
Height & $+/-6 \mathrm{kV}$ \\
Radii & \\
Voltage & $100 \mathrm{~mm}$ \\
& $25 \mathrm{~mm}$ \\
Quadrupoles & $+/-1 \mathrm{kV}$ \\
\hline Length & and $265 \mathrm{~mm}$ \\
Aperture Radius & \\
Voltage & \\
\hline \hline
\end{tabular}

\section{OUTLOOK}

In order to study all the different elements necessary for a complete ring, a quarter ring section has been build up in Frankfurt, Fig. 7. Special focus was put on practical aspects.

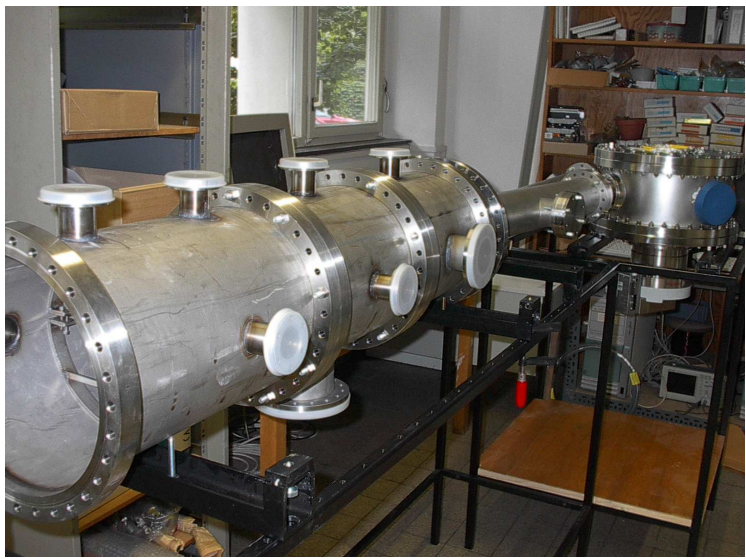

Figure 7: Overview of the quarter ring section

At present, vacuum measurements are made with these cavities and the next step will be to test the diagnostic [4] and optical elements with a test beam.

\section{ACKNOWLEDGEMENTS}

The authors would like to thank Søren Pape Møller for the many discussions and fruitful cooperation nihundrede tak for Hjaelp !

\section{REFERENCES}

[1] S.P. Møller, "ELISA, an electrostatic storage ring for atomic physics"

NIM A 394, 281 (1997)

[2] C.P. Welsch, "An electrostatic storage ring at IAP" PAC, Chicago, IL, USA, 2001

[3] C.P. Welsch et al, „Analysis of Field Perturbation due to Electrode Errors in an Electrostatic Storage Ring" EPAC, Paris, France, 2002

[4] C.P. Welsch et al, „A Flexible Control System for an Electrostatic Storage Ring" EPAC, Paris, France, 2002 